\title{
A novel staining method for quantification and 3D visualisation of capillaries and muscle fibres
}

\author{
V. Čebašek, ${ }^{1}$ L. Kubínová, ${ }^{2}$ S. Ribarič, ${ }^{3}$ I. Eržen ${ }^{1}$
}

${ }^{1}$ Institute of Anatomy, Medical faculty, University of Ljubljana, Ljubljana, Slovenia, ${ }^{2}$ Institute of

Physiology, Academy of Sciences of the Czech Republic, Prague, Czech Republic; ${ }^{3}$ Institute of

Pathophysiology, Medical Faculty, University of Ljubljana, Ljubljana, Slovenia

(C)2004, European Journal of Histochemistry

The aim of this study was to introduce a combined fluorescent staining that clearly demonstrates capillaries and distinguishes them from the basal lamina of muscle fibres in skeletal muscle tissue. The triple staining with CD31, Griffonia (Bandeira) simplicifolia lectin (GSL I) and laminin efficiently distinguishes vascular endothelium from the basal lamina of skeletal muscle fibres in physiological and pathological conditions. The presented triple staining method has several advantages, which facilitate quantitative analysis of the capillary network, and its relation to individual muscle fibres.

Key words: 3D measurement, capillaries, confocal microscopy, immunohistochemistry, lectin histochemistry, skeletal muscle.

Correspondence: V. Čebašek, Institute of Anatomy, Medical faculty, University of Ljubljana, Korytkova 2, 1000, Ljubljana, Slovenia. Phone: international +386.1.543-7309. Fax: international +386.1.543-7301.

E-mail: vita.cebasek@mf.uni-lj.si

Paper accepted on October 13, 2003

European Journal of Histochemistry 2004; vol. 48 issue 2 [Apr-Jun]: 151-158
( apillaries are essential for the survival, normal function and adaptation of muscle tissue to changing workloads (Hudlicka 1991, Green et al. 1999). Traditionally, the morphometric characteristics of capillary network were measured in two dimensions (2D) from transverse or longitudinal tissue sections (Mathieu et al. 1983; Egginton and Ross, 1989).

The morphological analysis in 2D gives only a general description of the capillary bed since the capillaries form a network in three dimensions (3D). The precise topology and course of capillary network can only be described by 3D analysis of muscle tissue capillaries. Therefore, visualisation and analysis of capillaries in 3D are the methods of choice for detection of the early morphological changes in the capillary bed.

Clear visualization of capillaries is a prerequisite for quantitative analysis. Detailed studies of skeletal muscle capillary network are conventionally made from thin tissue sections. Histological methods like haematoxylin eosin or toluidine blue staining (Kiernan JA, 1990) require the presence of erythrocytes in the vessel lumen. Consequently, capillaries are often poorly recognisable because of erythrocytes being scarcely distributed in capillaries of skeletal muscle (Damon and Duling, 1984). Enzyme-histochemical methods like alkaline phosphatase (Gomori, 1941), dipeptidyl peptidase IV (Lojda, 1979), amylase periodic acid Schiff (PAS) reaction (Andersen, 1975) and ATPase (Sillau and Banchero, 1977; Rosenblat et al., 1987) visualise the enzyme product in the capillary wall. Although identification of capillaries is superior when compared to histological methods, the enzyme based capillary staining can provide inconsistent results due to different enzyme activity in control and pathological conditions (Hansen Smith et al., 1992b). Capillary wall was successfully and comparably visualised immuno-histochemically with antibodies against endothelium (von Willebrand factor 
(vWF), CD31 (JC70A), CD34 (QBEnd 10) (Mukai et al., 1980; Delisser et al., 1993; Kuzu et al., 1992) or antibodies against basement membrane (fibronectin, collagen IV, laminin) (Barsky et al., 1983; Otsuki et al., 1990; Madsen and Holmskov, 1995). Different lectin-histochemical methods have also been proposed as good capillary staining techniques. Ulex europaeus agglutinin I lectin (UEA-I) proved to be a more sensitive and a more reliable human endothelium marker than vWF (Sehested and HouJensen, 1981). Griffonia (Bandeira) simplicifolia I lectin (GSI lectin) selectively binds to capillaries in rat skeletal muscle (Hansen Smith et al., 1988; Smolkova et al., 2001) and identifies both perfused and nonperfused microvessels (Greene et al., 1990). The advantage of GSI lectin over enzyme histochemical techniques is that binding of GSI lectin remains unchanged after exposure to hypoxia (Hansen Smith et al., 1992b). Therefore, it was proposed as a suitable marker of capillaries in physiological studies of skeletal muscle (Hansen Smith et al., 1992a).

The most often used methods for the quantification require identification of capillary profiles in stained thin tissue sections. The parameters describing the capillary frequency are number of capillaries per unit section area, number of capillaries per fibre number, diffusion distance, sharing factor, etc (Snyder, 1987; Egginton and Ross, 1989; Bennett et al., 1989). However, the whole information is restricted to two dimensions only, while the third dimension of the capillary geometry is lost.

In our previous paper (Kubínová et al., 2001), we introduced a stereological method for three dimensional (3D) quantification of capillaries in skeletal muscle. The capillaries were visualised by immunofluorescent detection of fibronectin (Eržen and Maravic, 1992). Digital images of successive optical sections, were obtained by confocal microscope from intact volume of thick tissue sections. Capillaries were visible deep into the tissue section, but they were not clearly distinguished from other structures in skeletal muscle. Recently, we have been looking for a staining technique that would further improve the identification of capillaries. Disadvantages of the capillary detection based on fibronectin staining are the following: (i) it is not sufficiently specific because additionally to the basal lamina of capillaries it stains also the basal lamina of muscle fibres and the loose connective tissue among muscle fibres (Stenman and Vaheri 1978;
Sanes JR, 1994) and (ii) in experimental or pathological conditions which favour growth of connective tissue, e.g. after denervation and reinnervation (Salonen et al., 1985; Siironen et al., 1992), the extensive and possibly also overstained fibronectin could mask capillary profiles in the endomysium.

The aim of this study was to develop an improved staining method for quantification of capillaries in 3D. Such a method must fulfill the following conditions: (i) capillaries must be clearly distinguished from other structures in skeletal muscle, (ii) capillaries should be visualised with fluorochromes to enable confocal focusing through thick sections, (iii) antibodies should penetrate sufficiently deep into the tissue section, (iv) preparation of specimens must be handy and fast enough for processing of large numbers of muscle samples at once, and ( $v$ ) the new staining should be suitable for the application of our previously developed stereological method. The triple staining with CD31, Griffonia (Bandeira) simplicifolia lectin (GSL I) and laminin seems to satisfy our requirements. To check the efficiency of this staining, we estimated capillary length to adjacent fibre length and compared the obtained results with our previous study, accomplished on fibronectin stained muscle sections (Kubínová et al., 2001).

\section{Materials and Methods}

All experimental work was performed on Wistar rats weighing between 200 to $250 \mathrm{~g}$.

\section{Tissue pretreatment}

Whole soleus (SOL) muscles were excised, placed in OCT embedding medium (Tissue-Tek), frozen in liquid nitrogen and stored at $-80^{\circ} \mathrm{C}$. Additionally, some muscles were fixed in cold acetone $\left(-20^{\circ} \mathrm{C}\right)$ overnight at $4^{\circ} \mathrm{C}$.

\section{Preparation of tissue slices}

The midsection of frozen muscles was cut with a cryostate (Reichert-Jung Frigocut 2800 ) at $-20^{\circ} \mathrm{C}$ into $30 \mu \mathrm{m}, 35 \mu \mathrm{m}, 40 \mu \mathrm{m}, 50 \mu \mathrm{m}$ or $60 \mu \mathrm{m}$ transverse sections. Sections were collected in PBS and subsequently mounted on ChemMate capillary Gap Microscope Slides previously coated with an adhesive (DAKO No. S 2024). They were air-dried and subsequently stored at $-80^{\circ} \mathrm{C}$.

Muscles fixed in acetone were cut with a razor blade into approximately $1 \mathrm{~mm}$ thick tissue sections and washed in PBS. 


\section{Staining protocols for capillaries and muscle fibres}

Cryosections of fresh frozen muscles were fixed in cold acetone $\left(-20^{\circ} \mathrm{C}\right)$ for 10 minutes and washed with phosphate-buffered saline $(0.01 \mathrm{M}, \mathrm{pH} 7.4)$ with $0.2 \%$ Triton X-100 (PBS-T) for 20 minutes. After preincubation in normal goat serum ( $1: 5$ in PBS) for $30 \mathrm{~min}$, to reduce background staining, sections were incubated in succession, with two different primary antibodies. The first antibody against CD31 (BD PharMingen No. 555025) (1:3000 in PBS-T) (at $4^{\circ} \mathrm{C}$ for 3 days) was used to label endothelial cells in capillaries. After several washes in PBS-T Alexa Fluor 488 (Molecular Probes A-11001) (1:350) with addition of $3 \%$ normal rat blocking serum was applied for 2 hours to visualize endothelium in green colour. The second antibody against laminin (CHEMICON No. MAB 1920) ( $1: 1000$ in PBS-T) was applied for 3 days at $4^{\circ} \mathrm{C}$ to label the basal lamina of muscle fibres. After excessive washing (in PBS-T) Alexa Fluor 546 (Molecular Probes A11003) at dilution 1:350 was applied for 2 hours to visualise basal laminas in red colour.

After thorough washing in PBS-T, sections were incubated overnight with fluorescein labelled Griffonia (Bandeira) simplicifolia lectin (GSL I) (Vector Laboratories No. FL 1101 (1:300, at $4^{\circ} \mathrm{C}$ ), an additional marker of capillaries.

Muscles fixed in acetone were stained floating in the incubation medium. Incubation time for each antibody was adjusted to the section thickness: from 3-6 days for CD31 and laminin and from 24 hours to 3 days for lectin. Secondary antibodies were applied for 2 days.

Negative controls were processed in the same way, only that the primary antibodies were replaced with PBS-T.

\section{Mounting}

After staining, all tissue sections were mounted in an aqueous mounting medium, a mixture of $10 \mathrm{~g}$ polyvinyl alcohol (PVA), $30 \mathrm{~mL}$ of glycerol and 70 $\mathrm{ml}$ of phosphate-buffered saline (PBS) ( $\mathrm{pH} 7.2$ ), with addition of small amount of sodium azide (NaN3). When mounting $1 \mathrm{~mm}$ thick tissue sections, silicone spacers were placed between the glass slides and glass coverslips (Molecular Probes P18175).

\section{Sampling for stereological measurements}

Triple stained $35 \mu \mathrm{m}$ thick transverse sections were selected for stereological measurements. Two sections from the right soleus muscle were obtained from five different rats. On average five fields per muscle were sampled by systematic uniform random sampling. A stack of 21 optical sections, $1 \mathrm{~mm}$ apart, was captured from every field by the twochannel Zeiss LSM 510 confocal microscope using a Zeiss Plan-Neofluar oil immersion 40x objective (numerical aperture 1.3).

Green and red fluorescences were excited with argon (488nm) and $\mathrm{He} / \mathrm{Ne}(543 \mathrm{~nm})$ laser. Emission signal was filtered using a narrow band BP 505$530 \mathrm{~nm}$ and LP $560 \mathrm{~nm}$ filter, respectively. From each stack of digitized images on average 11 fibres were chosen within an unbiased sampling frame (Gundersen, 1977).

\section{Stereological measurements}

Captured images with $x-y$ dimensions of $326 \mu \mathrm{m}$ by $326 \mu \mathrm{m}$ were analysed with the SLICER and FAKIR modules (Kubínová et al., 2001; Kubínová et al., 2002), running in Ellipse software environment (ViDiTo, Slovakia), for estimating length density of capillaries and fibre surface area. The results were expressed as the length of capillaries adjacent to a fibre within a $20 \mu \mathrm{m}$ muscle section (Lcap) $[\mu \mathrm{m}]$, as capillary length per adjacent fibre length ( Lcap/Lfib) $[\mu \mathrm{m} / \mu \mathrm{m}]$ and capillary length adjacent fibre surface area (Lcap/Sfib) $\left[\mu \mathrm{m} / \mu \mathrm{m}^{2}\right]$. Our previous results (Kubínová et al., 2001) were recalculated to a $20 \mu \mathrm{m}$ thick muscle section to enable easier comparison.

\section{Statistics}

Mean and standard deviation of the mean were calculated for each parameter. The mean values obtained with the two staining methods were compared with the paired, two tailed Student's t-test. A $P$ value $<0.05$ was considered to be statistically significant.

\section{$3 D$ reconstruction}

3D reconstruction of capillaries and muscle fibres was made from triple stained, thick sections applying the maximum projection algorithm to the stacks of confocal images in green and red channel and subsequent merging of these two projections.

\section{Results}

The novel, triple fluorescent staining (CD31, laminin and lectin) provided a clear-cut distinction 


\section{Figure 1. Capillaries and muscle fibre out- lines in the rat soleus muscle a) basal lamina of mus- cle fibres and capil- laries stained with anti-laminin (red channel only), b) double staining of endothelium with anti-CD31 and with fluorescein labelled lectin (GSL I) (green channel only) and c) a merged two-chan- nel image. Bar: 50 $\mu \mathrm{m}$.}
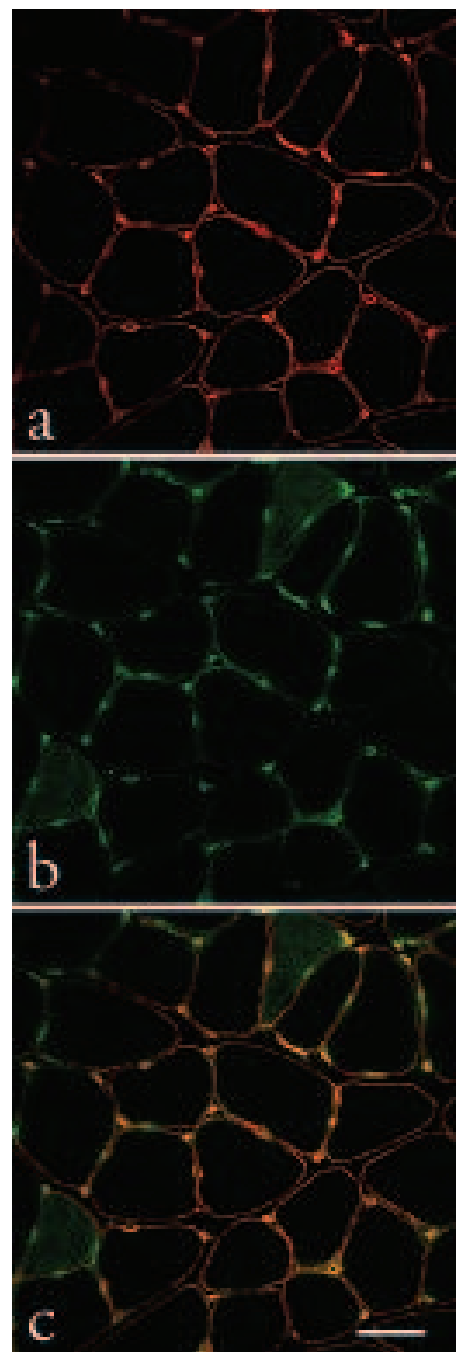

between capillaries and muscle fibres in rat soleus muscle (Figure 1). In the merged images from the two channels of the confocal microscope, green or yellow-green capillary profiles were in clear contrast with the red outlines of muscle fibres (Figure 1c). The interior of capillary profiles was labelled twice, with an anti-rat CD31 and with Griffonia (Bandeira) simplicifolia lectin (GSL I), both visualized with green fluourochrome (Figure lb). The basal lamina of capillaries and muscle fibres was labelled with an anti-rat laminin and visualized with a red fluorochrome (Figure 1a). Because of interference between green and red colour (i.e., collocalisation of antigens and emission interference of fluorochromes), capillary profiles in some places appear yellow (Figure 1c). Compared to the fibronectin staining the present triple staining evidently enhanced capillary identification. It took us about 5-10 min to measure the length of capillaries

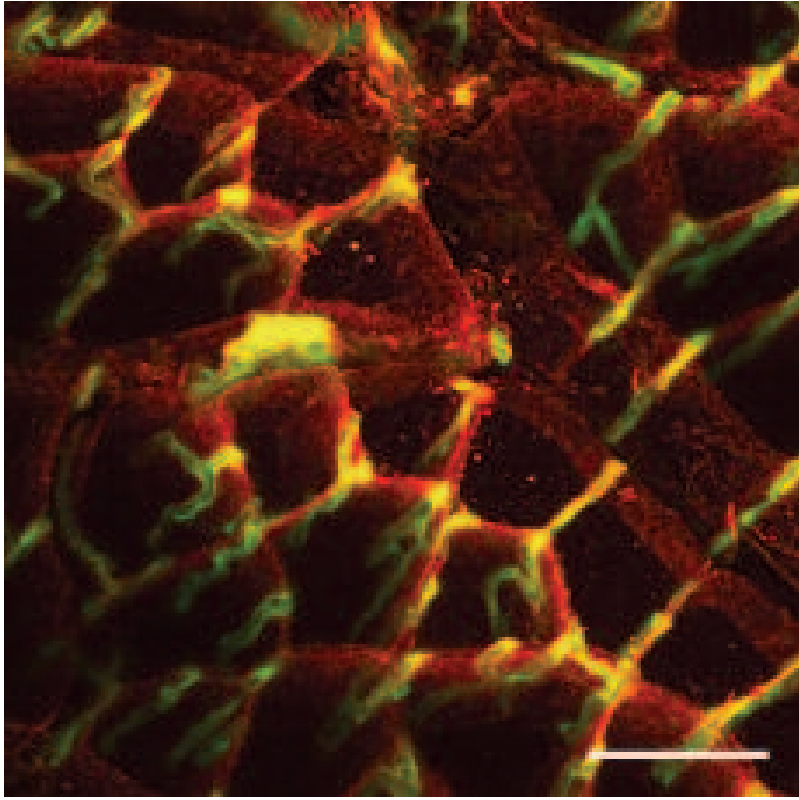

Figure 2. 3D reconstruction of the capillary network in the rat soleus muscle. Bar: 50 $\mathrm{mm}$.

adjacent to one muscle fibre and additional $4 \mathrm{~min}$ for measurements of the fibre surface area.

It was difficult to achieve successful mounting of $1 \mathrm{~mm}$ thick tissue sections in an upright position, although we used silicone spacers to make chambers between the cover slip and glass slide. However, staining was efficient and some samples were suitable for $3 \mathrm{D}$ reconstructions (Figure 2 ).

The most reliable results were obtained from 35 $\mu \mathrm{m}$ thick transverse sections. Consistent and good penetration of antibodies could be achieved to the depth of $20 \mu \mathrm{m}$ (Figure 3), penetration of antibodies, deeper than $30 \mu \mathrm{m}$, was inconsistent and insufficient.

Stereological analysis of the triple stained sections gave data comparable to the results of our previous study (Kubínová et al., 2001), where capillaries were stained with fibronectin (Table 1 ).

\section{Discussion}

In this study a novel, triple staining method was introduced for the detection of capillaries in skeletal muscle tissue. In merged images the staining provides green or yellow-green capillary profiles that are in clear contrast with the red outlines of muscle 

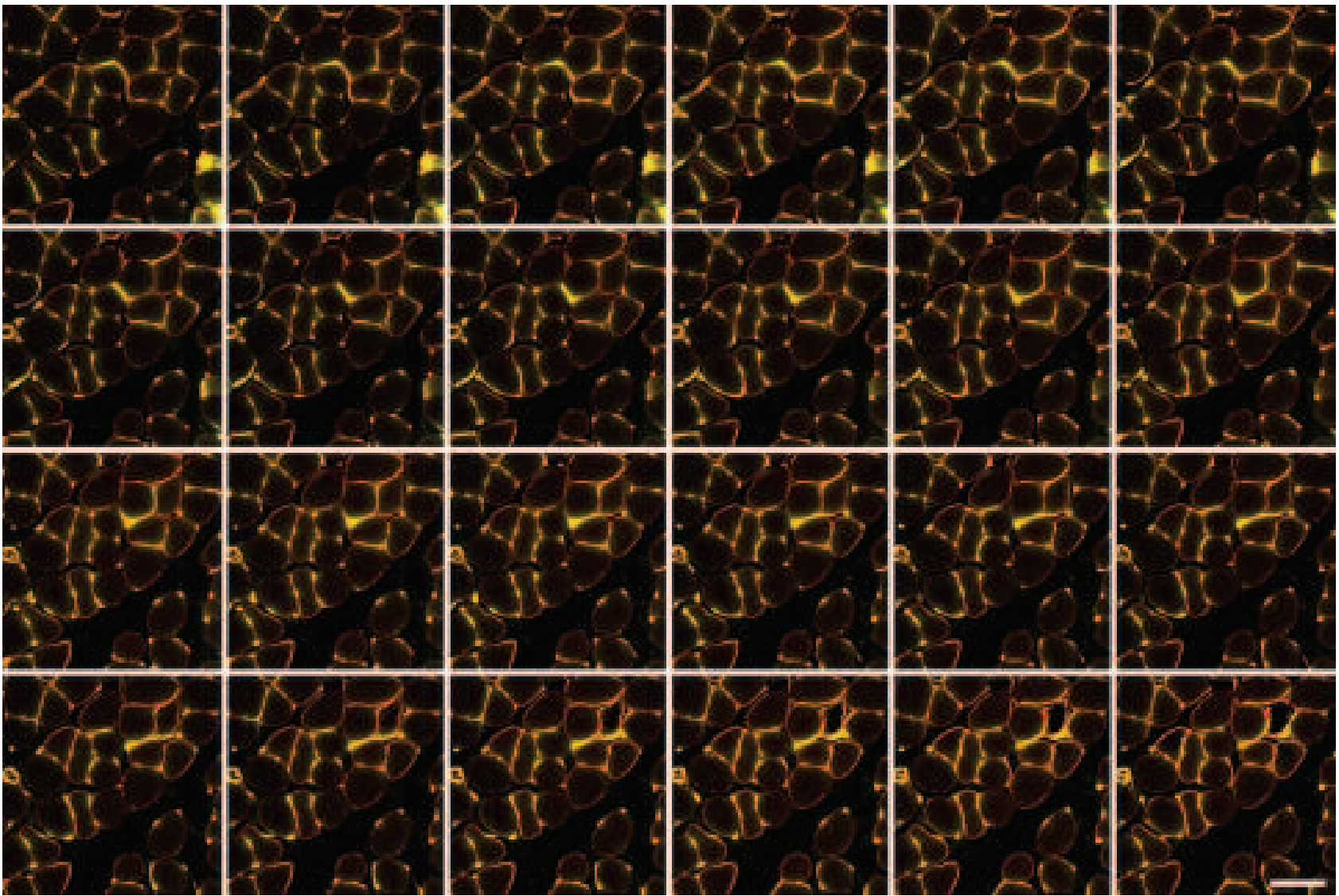

Figure 3. A stack of successive optical sections, $1 \mu \mathrm{m}$ apart, of the rat soleus muscle stained with CD31, Griffonia (Bandeira) simplicifolia lectin, and laminin. Bar: $100 \mu \mathrm{m}$.

Table 1. Morphometric characteristics of capillaries in the rat soleus muscle.

\begin{tabular}{lccc}
\hline \hline Method & Lcap & Lcap/Lfib & Lcap/Sfib \\
\hline Triple staining $^{\mathrm{a}}$ & $123,1 \pm 14$ & $6,130 \pm 0,878$ & $0,0272 \pm 0,0043$ \\
Fibronectin staining* $^{\mathrm{b}}$ & $115,4 \pm 22$ & $5,772 \pm 1,217$ & $0,0341 \pm 0,0083$ \\
a:b & NS & NS & NS \\
\hline
\end{tabular}

Lcap [ $\mu \mathrm{m}]$ - length of capillaries adjacent to a fibre within $20 \mu \mathrm{m}$ thick muscle sections; Lcap/Lfib [ $\mu \mathrm{m} / \mu \mathrm{m}]$ capillary length to adjacent fibre length; $L$ cap $/ \mathrm{Sfib}\left[\mu \mathrm{m} / \mu \mathrm{m}^{2}\right]$ capillary length to adjacent fibre surface area (mean $\pm S D$ ). Capillaries were identified with a triple staining (CD31, Griffonia (Bandeira) simplicifolia lectin, and laminin) and with fibronectin staining. *Results are adapted from Kubínová et al. (2001): NS= not significant

fibres. The staining has turned out to be superior to the fibronectin staining and, to our knowledge, to all other published staining techniques for the detection of capillaries in skeletal muscles. The advantages of the proposed staining are the following: (i) identification of capillaries and fibre outlines is quick and clear-cut; (ii) lectin binding to the capillary walls is in contrast to enzyme based stainings independent of hypoxia, which guarantees comparable staining under different experimental or pathological conditions; (iii) the staining distinguishes capillaries clearly from the surrounding tissue also in circumstances that favour growth of the connective tissue; (iv) the triple staining provides a reliable basis for potential semi-automatic and further automatic recognition of capillaries in skeletal muscle and ( $v$ ) faster identification of capillaries speeds up the quantitative analysis.

The thickness of transverse tissue sections that can be stained with this new triple staining method was limited by the depth of antibody penetration. If $50 \mu \mathrm{m}$ sections were incubated on slides, penetration of antibodies was incomplete. Several attempts to enhance penetration of antibodies into $50 \mu \mathrm{m}$ sections, like prolongation of the incubation time, addition of Triton X100 (Hartman et al., 1972) and dehydration with subsequent rehydration (Costa et al., 1980) were not successful enough. Therefore, we 
prepared $35 \mu \mathrm{m}$ thick sections where penetration of the antibodies and fluorescein labelled lectin was optimal. In some cases, the depth of the penetration of the antibody against laminin was only $20 \mu \mathrm{m}$, therefore the quantitative analysis was performed within the stacks of images not thicker than $20 \mu \mathrm{m}$.

We considered the possibility that capillary staining in large muscle samples or in whole muscles could be improved by injection of dyes. Injection of India ink and Microfil does not guarantee the visualisation of all vessels and capillaries (Plyley and Groom, 1975; Willner and Groom, 1977; Renkin et al., 1981), especially in skeletal muscle, where spatial and temporal heterogeneity of capillary perfusion was described (Lindbom, 1983; Lindbom and Arfors, 1984; Ellis et al., 1990). Further, the injection techniques are applicable only in muscles from experimental animals, but not for potential quantitative analysis of the capillary network in human biopsy samples, which is one of our future goals.

Many techniques have been developed to study microvascular network, however, only few of them allow 3D quantification of capillaries in skeletal muscle. The methods of vascular corrosion casting have been successfully applied for light (Cheung, 1996) and electron microscopic examination (Potter and Groom, 1983). The micro-corrosion cast technique using scanning electron microscopy enables visualisation of capillaries, however, morphometrical measurements are very time consuming. The corrosion casting procedures also dissolve the surrounding tissue of vessels, which precludes studies on the relationship between casts and surrounding tissues. Beside this, some of the casting materials are too viscous to fill microvascular bed of skeletal muscle and some of the muscles (e.g. m. gracilis) can be uniformly perfused only when the limb is left free to move (Potter and Groom, 1983).

For 3D quantification of capillaries, sections of soleus muscles hand cut into about $1 \mathrm{~mm}$ thick physical sections and processed floating in the incubation medium are an alternative to thinner sections cut with a cryotome and further processed on glass slides. Penetration of the antibodies and lectin into thick floating sections was superior to their penetration into sections incubated on glass slides. However, maintaining a constant orientation of the mounted muscle sections and focusing through the muscle tissue under the confocal microscope was more difficult than in the latter method.

When the relationships between different tissue constituents within intact tissues are analyzed, confocal microscopy presents a useful tool for quantitative measurements of their structure and arrangement in 3D. Digital images of perfectly registered stacks of serial optical sections from thick specimens that can be recorded by confocal microscope represent suitable data also for computer 3D reconstructions that can be made without the necessity to solve the tedious problem of alignment of images in successive physical sections (Pawley, 1995).

The results of the analysis and visualisation of density of the capillary network, obtained in this study, were comparable with our previous results in the rat soleus muscles (Kubínová et al., 2001). However, the capillaries were more clearly visualized, which facilitated our measurements of capillary length. The presented triple staining method reduced the time of measurement by more than $50 \%$, thus we are going to use it in our future studies of quantification and 3D visualization of capillaries and muscle fibres.

\section{Acknowledgments}

This study was supported by the Ministry of Science and Technology of Slovenia and the Ministry of Education, Youth and Sport of the Czech Republic (KONTAKT grant No. 001/2001) and by the Academy of Sciences of the Czech Republic (Grant AVOZ 5011922). Images were captured with the Zeiss LSM 510 microscope at the Institute of Pathophysiology, Medical Faculty of Ljubljana. The authors are very grateful to Majda Maasarani for her precious technical support and to Dr. Marko Kreft for his assistance with the confocal microscope.

\section{References}

Andersen P. Capillary density in skeletal muscle of man. Acta Physiol Scand 1975;95:203-5.

Barsky SH, Baker A, Siegal GP, Togo S, Liotta LA. Use of anti-basement membrane antibodies to distinguish blood vessel capillaries from lymphatic capillaries. Am J Surg Pathol 1983;7:667-77.

Bennett RA, Pittman RN, Sullivan SM. Capillary spatial pattern and muscle fiber geometry in three hamster striated muscles. Adv Exp Med Biol 1989;248:259-70.

Cheung LK. The blood supply of the human temporalis muscle: a vascular corrosion cast study. J Anat 1996;189:431-8.

Costa M, Buffa R, Furness JB, Solcia E. Immunohistochemical localization of polypeptides in peripheral autonomic nerves using whole mount preparations. Histochemistry 1980;65:157-65.

Damon $\mathrm{DH}$, Duling BR. Distribution of capilllary blood flow in the microcirculation of the hamster: an in vivo study using epifluorescent microscopy. Microvasc Res 1984;27:81-95.

Delisser HM, Newman PJ, Albelda SM. Platelet endothelial cell adhe- 
sion molecule (CD31). Curr Top Microbiol Immunol 1993;37-45.

Egginton S, Ross HF. Quantifying capillary distribution in four dimensions. Adv Exp Med Biol 1989;248:271-80.

Ellis CG, Wrigley SM, Potter RF, Groom AC. Temporal distributions of red cell supply rate to individual capillaries of resting skeletal muscle, in frog and rat. Int J Microcirc Clin Exp 1990;9:67-84.

Eržen I, Maravič V. Simultaneous histochemical demonstration of capillaries and muscle fibre types. Histochemistry 1992;99:57-60.

Gomori G. Distribution of acid phosphatase in tissues under normal and under pathologic conditions. Arch Pathol 1941;32:189-99.

Green H, Goreham C, Quyang J, Ball-Burnett M and Ranney D. Regulation of fibre size, oxidative potential and capillarization in human muscle by resistance exercise. Am J Physiol 1999; 276: R591-6.

Greene AS, Lombard JH, Cowley AW jr, Hansen-Smith FM. Microvessel changes in hypertension measured by Griffonia simplicifolial lectin. Hypertension 1990;15:779-83.

Gundersen HJG. Notes on the estimation of the numerical density of arbitrary profiles: the edge efect. J Microsc 1977;111:219-23.

Hansen-Smith F, Banker K, Morris L. Alternative histochemical markers for skeletal muscle capillaries: a statistical comparation among three muscles. Microvasc Res 1992a; 44:112-6.

Hansen-Smith FM, Blackwell LH, Joswiak GR. Expression of muscle capillary alkaline phosphatase is affected by hypoxia. J Appl Physiol 1992b; 73:776-80.

Hansen-Smith FM, Watson L, Lu DY, Goldstein I. Griffonia Simplicifolia I: fluorescent tracer for microcirculatory vessels in nonperfused thin muscles and sectioned muscle. Microvasc Res 1988; 36:199-215.

Hartman BK, Zide D, Udenfriend S. The use of dopamine-hydroxylase as a marker for the central noradrenergic nervous system in rat brain. Proc Natl Acad Sci USA 1972; 69:2722-6.

Hudlicka 0 What makes blood vessels grow? J Physiol 1991; 444:124.

Kiernan JA. Histological Staining in One or Two Colours. In: Kiernan JA, editor. Histological \& Histochemical Methods. Theory and practice, second edition. Pergamon Press, 0xford: 1990. p. 90-102.

Kubínová L, Janáček J, Ribarič $S$, Cebašek $V$, Eržen I. Three-dimensional study of the capillary supply of skeletal muscle fibres using confocal microscopy. J Muscle Res Cell Motil 2001;22:217-27.

Kubínová L, Janáček J, Krekule I. Stereological methods for estimating geometrical parameters of microscopical structure studied by threedimensional microscopical techniques. In: Diaspro A, Ed. Confocal and Two-photon Microscopy. Wiley-Liss: New York; 2002. p. 299332.

Kuzu I, Bicknell R, Harris AL, Jones M, Gatter KC, Mason DY. Heterogeneity of vascular endothelial cells with relevance to diagnosis of vascular tumours. J Clin Pathol 1992;45:143-8.

Lindbom L. Microvascular blood flow distribution in skeletal muscle. An intravital microscopic study in the rabbit. Acta Physiol Scand 1983; 525:1-40.

Lindbom L, Arfors KE. Non-homogeneous blood flow distribution in the rabbit tenuissimus muscle. Differential contropl of total flow and capillary perfusion. Acta Physiol Scand 1984;122:225-33.
Lojda Z. Studies on dipeptidyl (Amino) Peptidase IV (Glycyl-Proline Naphthylamidase). Histochemistry 1979;59:153-66.

Madsen K, Holmskov U. Capillary density measurements in skeletal muscle using immunohistochemical staining with anticollagen type IV antibodies. Eur J Appl Physiol 1995;71:472-4.

Mathieu 0, Cruz-Orive LM, Hoppeler H, Weibel ER. Estimating length density and quantifying anisotropy in skeletal muscle capillaries. $J$ Microsc 1983;131:31-46.

Mukai K, Rosai J, Burgdirf WHC. Localization of factor-VIII related antigen in vascular endothelial cells using immunoperoxidase method. Am J Surg Pathol 1980;4:273-6.

Otsuki $Y$, Kubo $H$, Magari S. Immunohistochemical differentiation between lymphatic vessels and blood vessels- use of anti-basement membrane antibodies and anti-factor VIII-related antigen. Arch Histol Cytol 1990;53 Suppl:95-105.

Pawley JB. Handbook of Biological Confocal Microscopy. Second Edition. Plenum Press: New York; 2002.

Plyley MJ, Groom AC. Geometrical distribution of capillaries in mammalian striated muscle. Am J Physiol 1975;228:1376-83.

Potter RF, Groom AC. Capillary Diameter and Geometry in Cardiac and Skeletal Muscle Studied by Means of Corrosion Casts. Microvasc Res 1983;25:668-84.

Renkin EM, Gray SD, Dodd LR. Filling of microcirculation in skeletal muscles during timed India ink perfusion. Am J Physiol 1981; 241:H174-86.

Rosenblat JD, Kuzon WM, Plyley MJ Jr, Pynn BR, McKee NH. A histochemical method for the simultaneous demonstration of capillaries and fiber type in skeletal muscle. Stain Technol 1987;62:85-92.

Salonen V, Lehto M, Kalimo M, Penttinen R, Aro H. Changes in intramuscular collagen and fibronectin in denervation atrophy. Muscle Nerve 1985;8:125-31.

Sanes JR. The Extracellular Matrix. In: Engel AC, Franzini-Armstrong $C$, eds. Myology basic and clinical, second edition, vol 1. McGrawHill: INC New York; 1994. p. 242-60.

Sehested M, Hou-Jensen K. Factor VIII related antigen as an endothelial cell marker in benign and malignant diseases. Virchows Arch 1981;391:217-25.

Siironen J, Sandberg M, Vuorinen V, Roytta M. Expression of type I and III collagens and fibronectin after transection of rat sciatic nerve. Reinnervation compared with denervation. Lab Invest 1992;67:807.

Sillau AH, Banchero N. Visualization of capillaries in skeletal muscle by the ATPase reaction. Pflugers Arch 1977;369:269-71.

Smolkova 0, Zavadka A, Bankston P, Lutsyk A. Cellular heterogeneity of rat vascular endothelium as detected by HPA and GS I lectin-gold probes. Med Sci Monit 2001;7:659-68.

Snyder GK. Estimating diffusion distances in muscle. J Appl Physiol 1987;63:2154-8.

Stenman S, Vaheri A. Distribution of a major connective tissue protein, fibronectin in normal human tissues. J Exp Med 1978;147:105464.

Willner LA, Groom AC. Inadequacy of filling the capillary bed of skeletal muscle with Microfil. Fed Proc 1977;36:523. 
V. Čebašek et al. 